\title{
Pacific Rim redux: lorlatinib, the ultimate Jaeger?
}

\author{
Viola W. Zhu, Sai-Hong Ignatius Ou \\ Chao Family Comprehensive Cancer Center, Division of Hematology/Oncology, Department of Medicine, University of California, Irvine School of \\ Medicine, Orange, CA, USA \\ Correspondence to: Viola W. Zhu, MD, PhD. Chao Family Comprehensive Cancer Center, Division of Hematology/Oncology, Department of Medicine, \\ University of California, Irvine School of Medicine, 101 The City Dr S, Bldg 56, Room 244, Orange, CA 92868, USA. Email: zhuvw@uci.edu. \\ Provenance: This is an invited Editorial commissioned by Section Editor Jin Kang [Division of Pulmonary Oncology, Cancer Center, Guangdong \\ Lung Cancer Institute (GLCI), Guangdong General Hospital (GGH), Guangzhou, China]. \\ Comment on: Yoda S, Lin JJ, Lawrence MS, et al. Sequential ALK Inhibitors Can Select for Lorlatinib-Resistant Compound ALK Mutations in ALK- \\ Positive Lung Cancer. Cancer Discov 2018;8:714-29.
}

Submitted Sep 11, 2018. Accepted for publication Sep 22, 2018.

doi: $10.21037 / \mathrm{atm} .2018 .09 .49$

View this article at: http://dx.doi.org/10.21037/atm.2018.09.49

Lorlatinib is the most potent anaplastic lymphoma kinase (ALK) inhibitor to date based on pre-clinical models and also has excellent central nervous system penetration with an efflux/influx ratio of 1.5 (1). Emerging phase $1 / 2$ data have demonstrated its efficacy in patients after disease progression on first- and second-generation ALK inhibitors (2). In the era of targeted therapy, it is generally accepted that the best treatment should be offered upfront. Until the phase 3 CROWN trial (NCT03052608) that compares lorlatinib with crizotinib in metastatic treatmentnaïve patients with $A L K$-rearranged non-small cell lung cancer (NSCLC) reads out in a few years, is there evidence to support using lorlatinib upfront? Yoda et al. may have provided the scientific argument for this approach (3).

The group aimed to explore mechanisms of resistance to lorlatinib. First, they utilized $\mathrm{Ba} / \mathrm{F} 3$ cell models expressing non-mutant or mutant EML4-ALK to mimic treatmentnaïve or ALK inhibitor (first- and second-generation)resistant clinical setting. These cells were mutagenized with $N$-ethyl- $N$-nitrosourea (ENU), a potent chemical to induce point mutations. As authors pointed out, one limitation of ENU mutagenesis is that it is known to induce certain single-nucleotide substitutions (GC to AT, AT to GC, and AT to TA). Nevertheless, they showed that treatment with 300-600 $\mathrm{nM}$ of lorlatinib successfully suppressed all single ALK mutations including the solvent-front mutation G1202R. Further ENU mutagenesis screening of Ba/F3 cells harboring single ALK mutations (C1156Y, F1174C, L1196M, G1202R, and G1269A) was performed to generate
24 different compound mutations resistant to lorlatinib. To confirm these compound mutations indeed confer resistance to lorlatinib, Ba/F3 cell lines expressing ALK L1196M/ L1198F, ALK G1202R/L1196M, and ALK G1202R/ L1198F were created. Based on the cell viability assay, the ALK G1202R/L1196M double mutation exhibited resistance to all ALK inhibitors whereas the ALK G1202R/ L1198F and ALK L1196M/L1198F double mutations were sensitive to crizotinib but resistant to second-generation ALK inhibitors and lorlatinib.

To validate clinically meaningful lorlatinib-resistant mutations, a total of 20 patients with $A L K$-rearranged NSCLC who had progressed on lorlatinib underwent repeat tissue biopsies. Out of eight patients with primary resistance (progressive disease as the best response) to lorlatinib, seven had no ALK mutation detected, suggesting ALK-independent (off-target) mechanisms of resistance. The remaining 12 patients had acquired resistance (stable disease or better $\geq 6$ months followed by progressive disease) to lorlatinib, among whom six compound mutations were detected. These are ALK C1156Y/L1198F (ALK C1156Y detected in pre-lorlatinib biopsy), ALK I1171N/ D1203N, ALK G1202R/L1196M (ALK G1202R detected in pre-lorlatinib biopsy), ALK G1202R/G1269A, ALK G1202R/L1204V/G1269A (ALK G1202R detected in prelorlatinib biopsy), and ALK E1210K/D1203N/G1269A (ALK E1210K/D1203N detected in pre-lorlatinib biopsy). Comparing pre- and post-lorlatinib mutation spectrum suggests that these resistance mutations developed in a 
stepwise fashion in response to selective pressure of more and more potent ALK inhibitors.

Authors subsequently selected three patients and performed whole exome sequencing (WES) on their serial biopsies in order to characterize clonal evolution. The first patient was treated with crizotinib, alectinib, and then lorlatinib. WES analysis demonstrated a dominant clone in treatment-naïve specimen followed by a crizotinib-resistant subclone, an alectinib-resistant subclone containing ALK G1202R, and lastly a lorlatinib-resistant subclone containing ALK G1202R/L1196M. Notably, this ALK G1202R/L1196M double mutation was also identified in ENU mutagenesis screening and chosen to be expressed in $\mathrm{Ba} / \mathrm{F} 3$ cells for the cell viability assay. The second patient was treated with sequential crizotinib, brigatinib, and lorlatinib. Clonal analysis showed a crizotinib-resistant clone containing ALK E1210K which gave rise to three brigatinib-resistant subclones containing ALK E1210K/ S1206C, ALK E1210K/D1203N, and ALK E1210K/ L1122V. The subclone containing the ALK E1210K/ D1203N double mutation eventually became dominant and acquired a third mutation G1269A after exposure to lorlatinib for over 15 months. The third patient was treated with crizotinib, alectinib, ceritinib, and lorlatinib but had primary resistance to lorlatinib. The clone containing ALK I1171N was seen after progression on alectinib which gave rise to a subclone containing ALK I1171N/L1198M conferring resistance to lorlatinib.

While WES and clonal analysis would be impractical for clinical management of patients each time they progress on an ALK inhibitor, the extensive and elegant work carried out by Yoda et al. clearly demonstrated how lorlatinib-resistant double or triple mutations had accumulated through sequential exposure to ALK inhibitors with increasing potency. As of right now, none of the ALK inhibitors even in development can overcome this mechanism of resistance. Systemic chemotherapy with or without an ALK inhibitor should be the next treatment option. To prevent emergence of these highly refractory compound mutations, one would strongly argue for the upfront use of lorlatinib.

As reported by Gainor et al. (4), single ALK resistance mutations were more commonly identified in patients treated with second-generation ALK inhibitors as compared with crizotinib (54\% for ceritinib, $53 \%$ for alectinib, and $71 \%$ for brigatinib versus $20 \%$ for crizotinib). Breaking down the specific ALK resistance mutations, the solventfront mutation G1202R accounted for approximately half of all the identified mutations. This work set the stage for the use of lorlatinib to rescue patients resistant to secondgeneration ALK inhibitors as pre-clinical models have demonstrated activities of lorlatinib against the broadest spectrum of single ALK resistance mutations including G1202R. In the US, alectinib is already replacing crizotinib in the front-line setting. The longer follow-up of the ALEX study was recently reported and median progressionfree survival (PFS) by investigator-assessment extended to 34.8 months for alectinib as compared with 10.9 months for crizotinib (5). Brigatinib is another potent secondgeneration ALK inhibitor. Based on the results of the ALTA study showing median PFS of 15.6 months by blinded independent central review (6), the longest median PFS among all second-generation ALK inhibitors in phase 2 trials (7), we anticipate the phase 3 ALTA-1L study comparing brigatinib with crizotinib (NCT02737501) would be significantly positive and thus gaining brigatinib its first-line indication very soon. By then, whether to use upfront alectinib or brigatinib followed by lorlatinib would be a dealer's choice and both drugs are well tolerated. Notably, lorlatinib has several unique adverse events (AEs) including hypercholesterolemia, hypertriglyceridemia, peripheral edema, peripheral neuropathy, and cognitive effects. In phase 1 study, most of these AEs were grade 1-2 and no patients had permanent discontinuation of lorlatinib due to these treatment-related AEs (8). Therefore, given its great potency, relative tolerability, and more importantly the potential to prevent stepwise accumulation of single, double, and triple ALK resistance mutations, lorlatinib should replace second-generation ALK inhibitors for treatment-naïve patients as soon as the CROWN study grants its first-line indication.

Finally, with the upfront use of lorlatinib, ALKindependent mechanisms of resistance are expected to emerge, including activation of bypass signaling pathways such as EGFR, IGF-1R, KIT, MET, RAS/MAPK, SHP2, and SRC, as well as EMT (epithelial-to-mesenchymal transition) and small cell transformation (4,9-16). As previously mentioned, Yoda et al. selected 20 patients who had progressed on lorlatinib for repeat tissue biopsies. No ALK mutations were detected in a total of 12 patients and they likely harbored off-target resistance mechanisms (3). If a bypass signaling pathway is upregulated and can be targeted by a small molecular inhibitor, one would imagine a combination strategy provided that there are no significant overlapping toxicities between this small molecular inhibitor and lorlatinib. Fortunately, most of these signaling pathways already have matched inhibitors that are either approved or 
in development to be readily tested in combination therapy. On the other hand, if the resistance mechanism is EMT or small cell transformation, systemic chemotherapy with or without an ALK inhibitor should be offered.

Undoubtedly, in defense against $A L K$-rearranged NSCLC, lorlatinib represents the ultimate Jaeger at the present moment but its Achilles' heel is compound ALK resistance mutations. Therefore, designing next-generation ALK inhibitors that can overcome these mutations is urgently needed.

\section{Acknowledgements}

None.

\section{Footnote}

Conflicts of Interest: VW Zhu has received honoraria from Roche-Foundation Medicine, Roche/Genentech, Takeda, and Biocept, and consulting fees from TP Therapeutics. SI Ou has received honoraria from Pfizer, Roche-Foundation Medicine, Roche/Genentech and Takeda, and has stock ownership in TP Therapeutics.

\section{References}

1. Johnson TW, Richardson PF, Bailey S, et al. Discovery of (10R)-7-amino-12-fluoro-2,10,16-trimethyl-15-oxo10,15,16,17-tetrahydro-2H-8,4-(metheno)pyrazolo[4,3-h] [2,5,11]-benzoxadiazacyclotetradecine-3-carbonitrile (PF06463922), a macrocyclic inhibitor of anaplastic lymphoma kinase (ALK) and c-ros oncogene 1 (ROS1) with preclinical brain exposure and broad-spectrum potency against ALKresistant mutations. J Med Chem 2014;57:4720-44.

2. Shaw AT, Martini JF, Besse B, et al. Abstract CT044: Efficacy of lorlatinib in patients (pts) with advanced ALKpositive non-small cell lung cancer (NSCLC) and ALK kinase domain mutations. Cancer Res 2018;78:Abstract nr CT044.

3. Yoda S, Lin JJ, Lawrence MS, et al. Sequential ALK inhibitors can select for lorlatinib-resistant compound ALK mutations in ALK-positive lung cancer. Cancer Discov 2018;8:714-29.

4. Gainor JF, Dardaei L, Yoda S, et al. Molecular mechanisms of resistance to first- and second-generation ALK inhibitors in ALK-rearranged lung cancer. Cancer Discov 2016;6:1118-33.

5. Camidge DR, Peters S, Mok T, et al. Updated efficacy and safety data from the global phase III ALEX study of alectinib (ALC) vs crizotinib (CZ) in untreated advanced ALK+ NSCLC. J Clin Oncol 2018;36:abstr 9043.

6. Kim DW, Tiseo M, Ahn MJ, et al. Brigatinib in Patients With Crizotinib-Refractory Anaplastic Lymphoma KinasePositive Non-Small-Cell Lung Cancer: A Randomized, Multicenter Phase II Trial. J Clin Oncol 2017;35:2490-8.

7. Ou SI. Further advances in the management of anaplastic lymphoma kinase-mutated non-small-cell lung cancer. J Clin Oncol 2017;35:2463-6.

8. Shaw AT, Felip E, Bauer TM, et al. Lorlatinib in nonsmall-cell lung cancer with ALK or ROS1 rearrangement: an international, multicentre, open-label, single-arm firstin-man phase 1 trial. Lancet Oncol 2017;18:1590-9.

9. Doebele RC, Pilling AB, Aisner DL, et al. Mechanisms of resistance to crizotinib in patients with ALK gene rearranged non-small cell lung cancer. Clin Cancer Res 2012;18:1472-82.

10. Katayama R, Shaw AT, Khan TM, et al. Mechanisms of acquired crizotinib resistance in ALK-rearranged lung Cancers. Sci Transl Med 2012;4:120ra17.

11. Lovly CM, McDonald NT, Chen H, et al. Rationale for co-targeting IGF-1R and ALK in ALK fusion-positive lung cancer. Nat Med 2014;20:1027-34.

12. Gouji T, Takashi S, Mitsuhiro T, et al. Crizotinib can overcome acquired resistance to $\mathrm{CH} 5424802$ : is amplification of the MET gene a key factor? J Thorac Oncol 2014;9:e27-8.

13. Hrustanovic G, Olivas V, Pazarentzos E, et al. RAS-MAPK dependence underlies a rational polytherapy strategy in EML4-ALK-positive lung cancer. Nat Med 2015;21:1038-47.

14. Dardaei L, Wang HQ, Singh M, et al. SHP2 inhibition restores sensitivity in ALK-rearranged non-small-cell lung cancer resistant to ALK inhibitors. Nat Med 2018;24:512-7.

15. Crystal AS, Shaw AT, Sequist LV, et al. Patient-derived models of acquired resistance can identify effective drug combinations for cancer. Science 2014;346:1480-6.

16. Ou SI, Lee TK, Young L, et al. Dual occurrence of ALK G1202R solvent front mutation and small cell lung cancer transformation as resistance mechanisms to second generation ALK inhibitors without prior exposure to crizotinib. Pitfall of solely relying on liquid re-biopsy? Lung Cancer 2017;106:110-4.

Cite this article as: Zhu VW, Ou SI. Pacific Rim redux: lorlatinib, the ultimate Jaeger? Ann Transl Med 2018;6(Suppl 1):S40. doi: 10.21037/atm.2018.09.49 